\title{
Reduction of image noise in high-pitch CT imaging of the abdomen using a first generation iterative reconstruction algorithm - a phantom study
}

\author{
Beeres Martin, Graf Thomas, Herrmann Eva, Bauer Ralf W, Kerl J Matthias, Schell Boris, \\ Siebenhandl Petra, Lee Clara, Gruber-Rouh Tatjana, Vogl Thomas J, Lehnert Thomas \\ Institute for Diagnostic and Interventional Radiology, Frankfurt, Germany. \\ Correspondence: Beeres Martin. Address: Institute for Diagnostic and Interventional Radiology, Theodor-Stern-Kai 7 \\ (House 23c) 60590 Frankfurt am Main Germany. Email: beeres@gmx.net
}

Received: September 25, 2014 Accepted: October 30, $2014 \quad$ Online Published: November 16, 2014

DOI : $10.5430 /$ ijdi.v2n1p29

URL: http://dx.doi.org/10.5430/ijdi.v2n1p29

\begin{abstract}
Purpose: Evaluation of different high-pitch dual-source settings and the influence of iterative reconstruction (IRIS) on image noise in abdominal CT-scanning of a phantom - feasible study.

Materials and Methods: The institutional review board has approved this study. To evaluate whether the dual-source high-pitch mode (Somatom Definition FLASH, Siemens) is feasible for abdominal imaging, we took an Alderson RANDO Phantom and reduced the pitch in 0.2 steps from 3.2 to 1.6. The Care Dose was switched on. The current tube voltage was $100 \mathrm{kV}$ in one group and $120 \mathrm{kV}$ in the other. The Ref.mAS for each scan was 210 for both groups. The images were reconstructed in slices/increments of $1 \mathrm{~mm} / 1 \mathrm{~mm}, 2 \mathrm{~mm} / 2 \mathrm{~mm}$ and $5 \mathrm{~mm} / 5 \mathrm{~mm}$ with and without IRIS [B30 kernel or I30 kernel (the IRIS kernel)]. In one defined slice position, we measured four defined and constant-sized ROIs, and evaluated the standard deviation as the image noise marker. To analyze the data statistically, we used a step-wise estimation approach for a linear model with interactions.

Results: IRIS significantly decreases the standard deviation as the image noise marker, especially in thin slices. The image noise was decreased by a factor of $0.716(p<0.001), 0.733(p<0.001)$ and $0.784(p<0.001)$ in $1 \mathrm{~mm}, 2 \mathrm{~mm}$ and $5 \mathrm{~mm}$ slices, respectively.

Conclusion: In our study, using IRIS we managed to reduce image noise significantly (all $p$-values $<0.001$ ) compared to the normal reconstruction mode, especially in thin slices (scans performed in $1 \mathrm{~mm}, 2 \mathrm{~mm}$ and $5 \mathrm{~mm}$ slices). We may therefore be able to reduce the radiation dose applied to patients whilst obtaining the same image quality using IRIS.
\end{abstract}

\section{Keywords}

Computer-tomography, Dual-source CT, High-pitch, Abdominal imaging

\section{I ntroduction}

CT imaging is nowadays widely and often used in clinical practice and there is still one remaining problem - the radiation dose ${ }^{[1]}$. Technical advantages have been made so the radiation dose has been remarkably decreased since the beginning of 
CT examinations in the 70's. The most important step for us remains the automatic tube-current modulation ${ }^{[2]}$. One promising new technique is the high-pitch imaging mode in dual source CT scanners ${ }^{[3,4]}$.

In the technical development of CT scanners, dual-source CT is currently one definition of state of the art computer tomography. The possibility of using two tubes to acquire simultaneous images constitutes a new technique that can be used in one of two ways. First, the advantages of two different tube current products - and therefore two different absorption spectra - can be exploited in dual energy $\mathrm{CT}^{[5,6]}$. Second, the two tubes can be operated with the same voltage and the table feed accelerated so that the pitch (table feed per collimation) can be increased even further ${ }^{[4]}$. In the conventional single-source CT, the pitch is normally limited to a maximum value of 1.5. If a higher pitch is required, it must be assumed that gaps will appear in the data set and that the data set can no longer be fully reconstructed. In dual-source mode, it is now possible to close the gaps using the second tube, enabling the pitch to be increased to 3.0 or even 3.4 in some cases without having to accept a loss of information. Motion artefacts are therefore reduced and, in some cases, the dose can be reduced considerably especially in cases where ECG-gating is required ${ }^{[3,7-11]}$.

In this study, we wanted to advance the idea of dual-source high-pitch CTs even further. To this end, we investigated an Alderson RANDO anthropomorphic phantom (Alderson Research Laboratories Inc. Stanford, CT) using a dual-source CT scanner (Somatom Definition FLASH, Siemens, Erlangen, Germany). Our objective was to determine whether it is possible to examine the abdomen of a patient in high-pitch mode and to find out how this affects image noise when used with two different voltages or when reconstructed using new reconstruction software (IRIS). The further aim was to determine the extent to which this impacts on the noise in the image and whether improvements can be made for the future.

\section{Material and methods}

The comparison was carried out using an experimental setup that remained constant throughout. The phantom was placed on the CT table, and no changes were made to this throughout the whole CT examinations. First of all, the abdomen part of the phantom was examined in the conventional single-source CT mode (mode 1, see Table 1). This was followed by a series of dual-source high-pitch CT examinations beginning at a pitch of 3.2. This value was reduced in 0.2 steps with every test, finishing with 1.6 in the final examination. The investigations were carried out in the same scan range, once with a tube voltage of $120 \mathrm{kV}$ and once with $100 \mathrm{kV}$ (modes 2/3, see Table 1).

Table 1. Examination parameters

\begin{tabular}{|c|c|}
\hline Mode 1 (Single-Source Normal Pitch) & $\begin{array}{l}\text { Single-source CT baseline imaging: } 120 \mathrm{kV} \text { tube voltage; } 210 \text { Ref.mAs using dose } \\
\text { modulation (CareDose 4D); collimation } 128 \mathrm{~mm} \times 0.6 \mathrm{~mm} \text {; pitch } 1.0 \text {; rotation time } 0.5 \\
\text { seconds. }\end{array}$ \\
\hline Mode 2 (Dual-Source High-Pitch) & $\begin{array}{l}2 \times 120 \mathrm{kV} \text { tube voltage, } 210 \text { Ref.mAs using dose modulation (CareDose } 4 \mathrm{D} \text { ). The pitch } \\
\text { factor was decreased from } 3.2 \text { to } 1.6 \text { in steps of } 0.2 \text {. (i.e. pitch 3.2, pitch } 3.0 \ldots \text { pitch } 1.6 \text { ). } \\
\text { Collimation } 2 \mathrm{~mm} \times 128 \mathrm{~mm} \times 0.6 \mathrm{~mm} \text {, rotation time } 0.28 \text { seconds. }\end{array}$ \\
\hline Mode 3 (Dual-Source High-Pitch) & $\begin{array}{l}2 \times 100 \mathrm{kV} \text { tube voltage, } 210 \text { Ref.mAs using dose modulation (CareDose 4D). The pitch } \\
\text { factor was decreased from } 3.2 \text { to } 1.6 \text { in steps of } 0.2 \text {. (i.e. pitch 3.2, pitch } 3.0 \ldots \text { pitch } 1.6 \text { ). } \\
\text { Collimation } 2 \mathrm{~mm} \times 128 \mathrm{~mm} \times 0.6 \mathrm{~mm} \text {, rotation time } 0.28 \text { seconds. }\end{array}$ \\
\hline
\end{tabular}

The examinations were performed without thermoluminescent dosimeters (TLD) as an initial approach to obtain a comparable signal-to-noise ratio. Regarding the temporal resolution of this acquisition, it is important that the two tubes and detectors are angled at 94 degrees in this dual source scanner. Since 180 degrees of projection data are required to reconstruct cross-sectional images, the projection data set is complete once the gantry has rotated 94 degrees. Since the rotation time of this new dual source CT-Scanner is 280 milliseconds, this results in an image acquisition time of 94/360 × 280 milliseconds $=73$ milliseconds. 
The images obtained were reconstructed in two different kernels: once in a conventional filtered back-projection recon kernel B30, as frequently used for the abdomen, and once in the iterative reconstruction kernel, using the IRIS-Software, called I30 (see Figure 1). In addition, various slice thicknesses were reconstructed, which were also evaluated according to the procedure described below. The images were assessed in slices/increments of $1 \mathrm{~mm} / 1 \mathrm{~mm}, 2 \mathrm{~mm} / 2 \mathrm{~mm}$ and $5 \mathrm{~mm} / 5$ mm, calculated once with recon kernel B30 and once with kernel I30.

The images were assessed using a Siemens Syngo Workstation (syngo VE32D, Siemens).

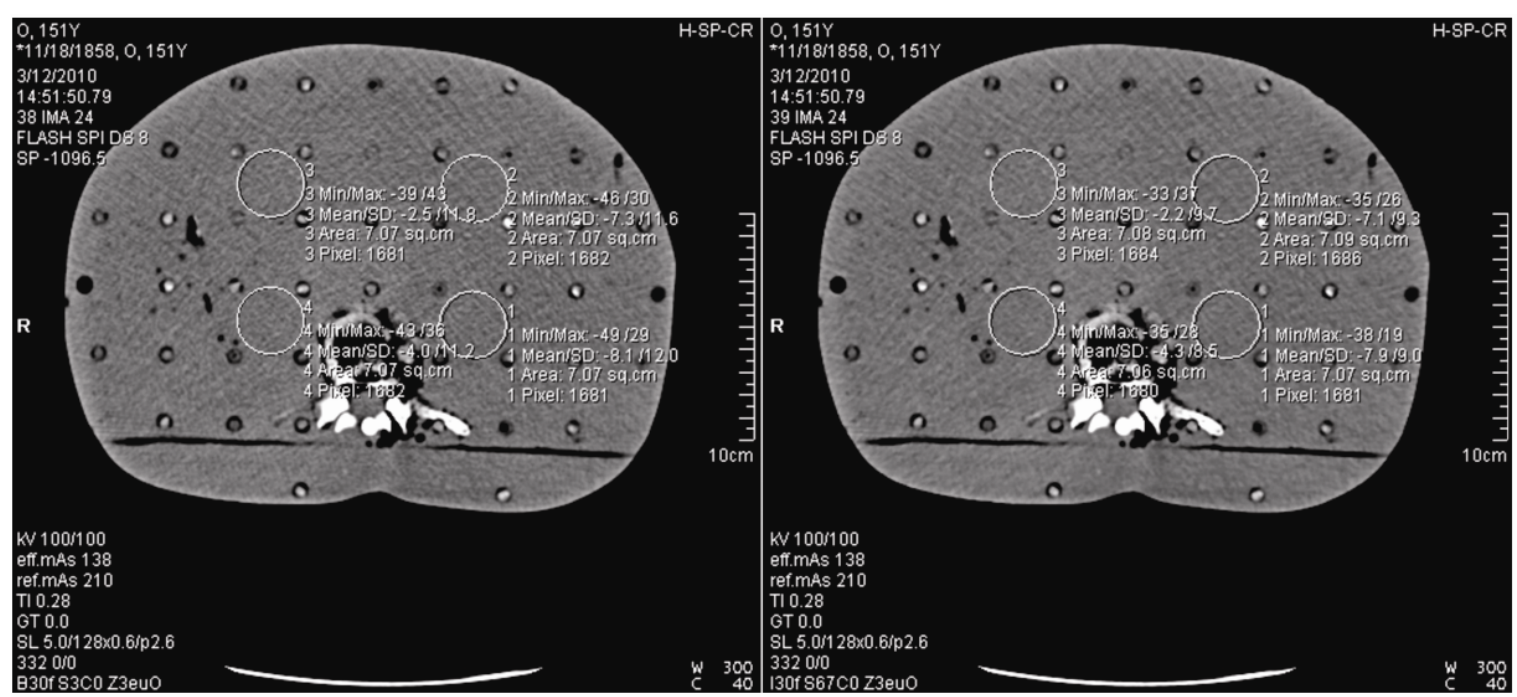

Figure 1. IRIS (right) vs. FBP (left)

\subsection{Measurements}

A region of interest (ROI) analysis was performed to determine and compare the noise in the phantom. The soft tissue of the abdomen was chosen for the main measurements, which were performed in four standardized $x-y$ positions. All measurements were performed for four different z-axis positions of the phantom with a standardized ROI size of $7 \mathrm{~cm}^{2}$. Image noise was defined as the standard deviation (SD) of HU values of the ROI, placed in a homogenous region.

\subsection{Statistical analysis}

The values were given as mean values of the standard deviation (mean +/- SD). The standard deviation was noted from each reading point and a mean value was computed from all standard deviations in a scan. A step-wise estimation approach for a linear model with interactions was used to analyze the data statistically. A commercially available software package was used for this (SPSS 15.0.1).

\section{Results}

First, it was shown that the noise in the images was considerably reduced using iterative reconstruction algorithms. In all of the reconstructed slice thicknesses, the standard deviation as a measure for image noise was reduced by a highly significant value of $p<0.001$. IRIS significantly decreases the standard deviation as marker of the image noise, especially in thin slices. The image noise was decreased by a factor of $0.733(p<0.001), 0.784(p<0.001)$ and $0.716(p<0.001)$ in $1 \mathrm{~mm}, 2 \mathrm{~mm}$ and $5 \mathrm{~mm}$ slices. See Figure 2 for an example of this.

The protocol called "conv." shows the "standard" taken in the single-source CT mode (120 kV; 210 Ref.mAs). It can generally be said that use of IRIS is able to bring about a significant reduction in the noise of a CT image. As we can also 
see from Figure 2, the difference in noise is particularly considerable in the thin slices. The thicker the slices are reconstructed, the less this has an impact on the existing statistical significance.

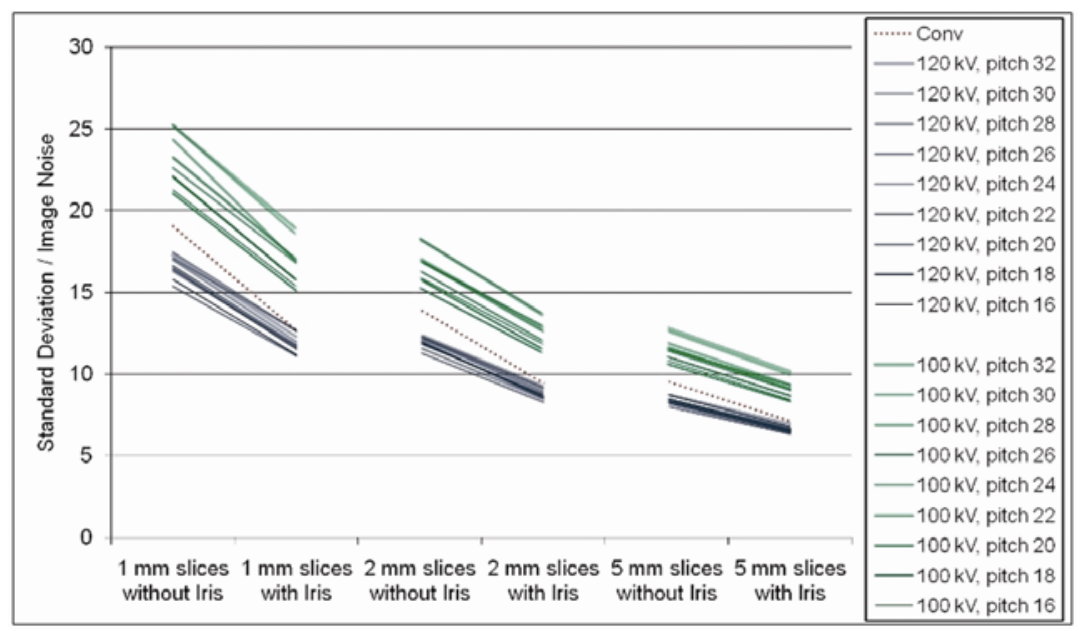

Figure 2. Image Noise IRIS vs. Non-IRIS Images

\section{Discussion}

In this experiment, we have shown that by using the iterative reconstruction software IRIS, noise can be reduced considerably, particularly if the CT data are reconstructed in thin slices. The investigations were performed on an Alderson phantom, once in the conventional single-source CT mode at $120 \mathrm{kV}$. This investigation served as the baseline for later comparisons. The same phantom in an unchanged position was subsequently examined in the dual-source high-pitch mode with a pitch between 3.2 and 1.6. The collimation remained constant at $0.6 \mathrm{~mm}$. The phantom was investigated once with $100 \mathrm{kV}$ and once with $120 \mathrm{kV}$. The position of the phantom and the scan range remained the same. The reconstructions were carried out once in the B30 kernel and once in the 130 kernel in $5 \mathrm{~mm}, 2 \mathrm{~mm}$ and $1 \mathrm{~mm}$ slices/increments. It emerged that use of IRIS causes a continual significant reduction of noise. There is a clear advantage in particular when images are reconstructed in thin slices $(1 \mathrm{~mm})$, which leads to an even greater reduction of noise than with thicker slices. In other words, use of IRIS can be particularly worthwhile in the reconstruction of thin slices. Nonetheless, reconstruction using IRIS takes slightly longer than with conventional recon, which could cause problems in the workflow. The time was not measured, however. It was merely noticed subjectively during the experiment that reconstruction with IRIS took longer than conventional filtered back-projection recon. Future research could involve investigating whether the dose in CT imaging can be reduced even further. Although the image noise would initially increase, it would be possible to use IRIS to reduce noise to a normal level to enable an overall reduction of the dose. Whether this would have to be in the abdomen is another question because the detector range of the second detector $(33 \mathrm{~cm})$ sometimes causes problems in the abdomen because the patient cannot be reproduced in full. Possibly IRIS could help to advance and improve imaging in high-pitch CT mode, particularly in tests where small details are important, such as in the region of a coronary artery or a petrosal bone.

We were able to show in our study that use of IRIS is advantageous with regard to image noise, particularly in thin-slice collimations

\section{References}

[1] Hall EJ, Brenner DJ. Cancer risks from diagnostic radiology. The British journal of radiology. 2008; 81: 362-378. PMid:18440940 http://dx.doi.org/10.1259/bjr/01948454 
[2] Kalender WA, Buchenau S, Deak P, et al. Technical approaches to the optimisation of CT. Phys Med. 2008; 24 : 71-79. PMid:18331808 http://dx.doi.org/10.1016/j.ejmp.2008.01.012

[3] Lell M, Marwan M, Schepis T, et al. Prospectively ECG-triggered high-pitch spiral acquisition for coronary CT angiography using dual source CT: technique and initial experience. European radiology. 2009; 19: 2576-2583. PMid:19760421 http://dx.doi.org/10.1007/s00330-009-1558-4

[4] Flohr TG, Leng S, Yu L, et al. Dual-source spiral CT with pitch up to 3.2 and 75 ms temporal resolution: image reconstruction and assessment of image quality. Medical physics. 2009; 36: 5641-5653. PMid:20095277 http://dx.doi.org/10.1118/1.3259739

[5] Bauer RW, Schulz JR, Zedler B, et al. Compound analysis of gallstones using dual energy computed tomography--results in a phantom model. European journal of radiology. 2010; 75: e74-80. PMid:19717259 http://dx.doi.org/10.1016/j.ejrad.2009.08.004

[6] Guimaraes LS, Fletcher JG, Harmsen WS, et al. Appropriate patient selection at abdominal dual-energy CT using $80 \mathrm{kV}$ : relationship between patient size, image noise, and image quality. Radiology. 2010; 257: 732-742. PMid:20959540 http://dx.doi.org/10.1148/radiol.10092016

[7] Goetti R, Baumuller S, Feuchtner G, et al. High-pitch dual-source CT angiography of the thoracic and abdominal aorta: is simultaneous coronary artery assessment possible? Ajr. 2010; 194: 938-944.

[8] Weustink AC, Neefjes LA, Kyrzopoulos S, et al. Impact of heart rate frequency and variability on radiation exposure, image quality, and diagnostic performance in dual-source spiral CT coronary angiography. Radiology. 2009; 253: 672-680. PMid:19864512 http://dx.doi.org/10.1148/radiol.2533090358

[9] Weustink AC, Mollet NR, Neefjes LA, et al. Preserved diagnostic performance of dual-source CT coronary angiography with reduced radiation exposure and cancer risk. Radiology. 2009; 252: 53-60. PMid:19451542 http://dx.doi.org/10.1148/radiol.2521082023

[10] Karlo C, Leschka S, Goetti RP, et al. High-pitch dual-source CT angiography of the aortic valve-aortic root complex without ECG-synchronization. European radiology. 2011. http://dx.doi.org/10.1007/s00330-010-1907-3

[11] Schell B, Bauer RW, Lehnert T, et al. Low-dose computed tomography of the paranasal sinus and facial skull using a high-pitch dual-source system-First clinical results. European radiology. 2010; 21: 107-112. PMid:20644936. http://dx.doi.org/10.1007/s00330-010-1892-6 